\title{
Efficacy of bilateral pallidotomy
}

\section{Richard Kim, M.D., M.S., Ron Alterman, M.D., Patrick J. Kelly, M.D., Enrico Fazzini, D.O., Ph.D, David Eidelberg, M.D., Ph.D., Alaksandar Beric, M.D., D.Sci., and Djorje Sterio, M.D.}

New York University Center for the Study and Treatment of Movement Disorders, New York, New York; Department of Neurosurgery, New York University Medical Center, New York, New York; and North Shore University Hospital, Manhassett, New York

Unilateral pallidotomy is a safe and effective treatment for medically refractory bradykinetic Parkinson's disease, especially in those patients with levodopa-induced dyskinesia and severe on-off fluctuations. The efficacy of bilateral pallidotomy is less certain.

The authors completed 11 of 12 attempted bilateral pallidotomies among 150 patients undergoing pallidotomy at New York University. In all but one patient, the pallidotomies were separated by at least 9 months. Patients were selected for bilateral pallidotomy if they exhibited bilateral rigidity, bradykinesia, or levodopa-induced dyskinesia prior to treatment or if they exhibited disease progression contralateral to their previously treated side.

The Unified Parkinson's Disease Rating Scale (UPDRS) and timed upper-extremity tasks of the Core Assessment Protocol for Intracerebral Transplantation (CAPIT) were administered to all 12 patients in the "off" state (12 hours without receiving medications) preoperatively and again at 6 and 12 months after each procedure. The median UPDRS and contralateral CAPIT scores improved $60 \%$ following the initial procedure ( $\mathrm{p}=0.008$, Wilcoxon rank sums test). The second pallidotomy generated only an additional 10\% improvement in the UPDRS and CAPIT scores ipsilateral to the original procedure $(\mathrm{p}=$ 0.05). Worsened speech was observed in two cases. In the 12th case, total speech arrest was noted during test stimulation. Speech returned within minutes after stimulation was halted. Lesioning was not performed.

These results indicate that bilateral pallidotomy has a narrow therapeutic window. Motor improvement ipsilateral to the first lesion leaves little room for further improvement from the second lesion and the risk of speech deficit is greatly enhanced. Chronic pallidal stimulation contralateral to a previously successful pallidotomy may prove to be a safer alternative for the subset of patients who require bilateral procedures.

\section{Key Words * Parkinson's disease * pallidotomy * movement disorders}

The recent reintroduction of posteroventral pallidotomy for the treatment of Parkinson's disease (PD) and refinements in its application have produced a body of experience that supports its use in patients who receive levodopa therapy but whose symptoms progress. $[2,4,5,9,10,12]$ Many issues remain to be 
clarified, however, such as the degree to which patients with different subtypes of PD respond, the optimal placement of lesions, and the role of pallidotomy relative to other surgical treatment options, such as thalamotomy and pallidal stimulation. One issue that will be of increasing importance as greater numbers of patients with PD undergo pallidotomy is the role of bilateral pallidotomy. Few studies have addressed the efficacy and safety of this procedure when performed in either a staged or contemporaneous fashion.[8,9] In this report we describe our experience in 12 patients in whom staged bilateral pallidotomies were performed or attempted.

\section{CLINICAL MATERIAL AND METHODS}

\section{Preoperative Patient Assessment}

Details of the protocol for preoperative assessment are described elsewhere[1] and are summarized here. All patients undergoing pallidotomy were examined by a neurologist, a neurosurgeon, and in some cases, a neurophysiologist. All candidates demonstrated predominantly bradykinetic symptoms of PD with severe on medication-off medication fluctuations, as well as levodopa-induced dyskinesias. Symptoms were present to a significantly disabling degree bilaterally, although asymmetry was usually present. A magnetic resonance (MR) image was obtained in each patient to assess the degree of cortical atrophy, the presence of which is believed to increase the risk of intraoperative hemorrhage. Patients also undergo ${ }^{18}$ F-fluorodeoxyglucose utilization positron emission tomography (PET) scanning as well as 18F-fluoro-dopa binding PET.

On the day before surgery, the Unified Parkinson's Disease Rating Scale (UPDRS)[6] and the three upper-extremity timed motor tasks of the Core Assessment Protocol for Intracerebral Transplantation (CAPIT)[11] were administered to each surgical candidate, both while they were receiving medication and 12 hours after ceasing medication. The tests were repeated at 6 and 12 months following each procedure. All scores reported here are those obtained when the patient had ceased taking medications for 12 hours. Differences in the median pre- and postoperative scores were tested for statistical significance by the Wilcoxon rank-sums test. Neuropsychological testing was also performed, in the midmorning and while the patient was taking medication.

\section{Surgical Technique}

Pallidotomy was performed on awake patients who had not received medication for at least 12 hours. The Leksell G-frame was applied to the patient's head using local anesthetic and minimal propofol sedation. Anatomical localization of the lesioning target was performed using MR imaging guidance. The Leksell MR imaging localizer (Elekta Instruments, Inc., Stockholm, Sweden) was applied and a stereotactic MR image was obtained using a 1.5-tesla magnet (Siemens, Erlangen, Germany). The anterior commissure-posterior commissure (AC-PC) line was defined on an axial scout view and subsequent coplanar axial images of 3-mm thickness were obtained. Coronal $\mathrm{T}_{1}$-weighted images were then obtained perpendicular to the AC-PC line.

The images were transferred to the CASS computer system (Midco, San Diego, CA) via 9-track tape. A digitized Schaltenbrand and Bailey atlas[14] was used to custom fit the axial atlas slice located $0.5 \mathrm{~mm}$ superior to the AC-PC line. Leksell's coordinates for posteroventral pallidotomy[10] were used and are as follows: 2 to $3 \mathrm{~mm}$ anterior to the midcommissural point, 4 to $6 \mathrm{~mm}$ ventral to the AC-PC line, and 18 to $23 \mathrm{~mm}$ lateral to the midline. The AC-PC axial image was coregistered with the digitized atlas and used to determine the $\mathrm{x}$ and $\mathrm{y}$ coordinates for the presumptive target. The $\mathrm{z}$ coordinate was determined from 
the next ventral slice, which is typically 3 to $4 \mathrm{~mm}$ below the AC-PC line. The anteroposterior (AP) angle of approach was approximately 45 š to the orbitomeatal line. The lateral angle was selected on the coronal image to pass just lateral to the lateral ventricle. For each trajectory, impedance was recorded beginning $50 \mathrm{~mm}$ short of the target. Microelectrode recording was begun at the $-15-\mathrm{mm}$ point.

Microelectrode recording was performed with a 1- $\mu$ tungsten-tipped microelectrode advanced by a hydraulic microdriver. The details of the equipment we used and the electrophysiological properties of the internal and external segments of the globus pallidus (GP) have been published previously.[1,5]

After a satisfactory trajectory was identified, macroelectrode stimulation was initiated using a 1.1-mm stimulating/lesioning electrode with a 3-mm exposed tip (Radionics, Inc., Burlington, MA). Stimulation consisted of a $0.2-\mathrm{msec}$ biphasic wave at 5 and $50 \mathrm{~Hz}$, delivered at 0 to $10 \mathrm{~V}$. The electrode was advanced to the ventral border of globus pallidus internus (GPi) as defined by microelectrode recording. The motor threshold is defined as the voltage at which contralateral muscle contraction is stimulated; the visual threshold is defined as the voltage at which scotomata or phosphenes in the contralateral visual field are elicited. We used $2 \mathrm{~V}$ or $5 \mathrm{mAmp}$ as our motor threshold and $3 \mathrm{~V}$ or $12 \mathrm{mAmp}$ as our visual threshold.

After stimulation was used to determine safe motor and visual thresholds, a test lesion was performed. The electrode was warmed to $605 \check{C}$ for 20 seconds and the motor strength was tested. If no new motor deficits were detected we proceeded with lesioning.

Lesions were made by heating the electrode to $80 \check{\mathrm{S} C}$ for 60 seconds. After cooling to $395 \mathrm{~S}$, the electrode was backed up $2 \mathrm{~mm}$ and another lesion was made. Typically four to five lesions were made at 2-mm intervals along the trajectory spanning GPi, as determined by microelectrode recording. Motor strength and speech were checked after each lesion.

\section{Postoperative Follow-Up Care and Analysis}

A computerized tomography (CT) scan was obtained immediately following the procedure. Patients were observed overnight and discharged on the 1st or 2nd postoperative day. Magnetic resonance imaging was performed within the 1st postoperative week to evaluate lesion placement. Subsequently, patients were examined by a neurologist every 3 months for the 1st year, every 4 months for the 2 nd year, and then annually. At each visit, the UPDRS and upper-extremity CAPIT tests were administered. At 6 months and 1 year, neuropsychological testing and PET were repeated. In all patients except one, at least 9 months were allowed to elapse before performing the contralateral lesion. The Wilcoxon rank-sums test was applied to preoperative and postoperative CAPIT and UPDRS scores. A probability value of 0.05 or less was considered significant.

\section{Patient Questionnaire}

Patients were contacted by telephone and answered a brief questionnaire regarding their subjective views of the results of each pallidotomy procedure, and whether any of their symptoms were improved, worsened, or unchanged by each procedure. The symptoms queried were dyskinesia, bradykinesia, rigidity, tremor, difficulty with walking, and difficulty with speech. Patients were also asked to describe their overall satisfaction with each procedure.

\section{RESULTS}




\section{Efficacy of Staged Bilateral Pallidotomy}

Both the median UPDRS and contralateral CAPIT scores improved $60 \%$ following the initial procedure ( $\mathrm{p}=0.008$, Wilcoxon rank sums test), as shown in Fig. 1 and 2. Moreover, ipsilateral CAPIT scores improved an average of $15 \%(\mathrm{p}=0.05)$.

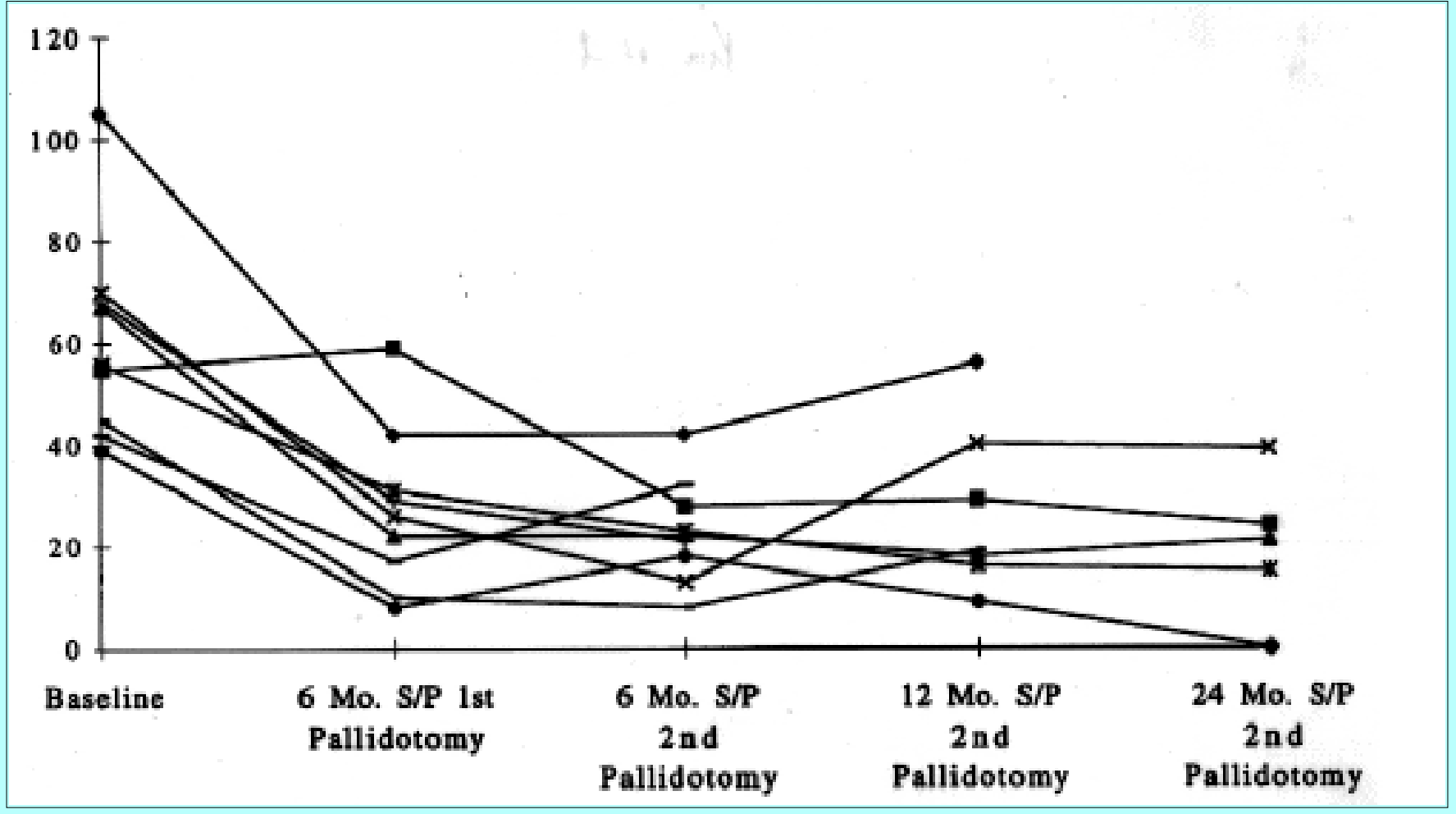

Fig. 1. Graph showing UPDRS scores following staged bilateral pallidotomy in eight patients. $\mathrm{SP}=$ status post.

The second pallidotomy produced an additional 10\% improvement in the UPDRS and CAPIT scores ipsilateral to the original procedure $(\mathrm{p}=0.05)$. This effect was stable over the follow-up period of 24 months after the second procedure. The effects on both the activities of daily living and motor scores of the UPDRS were similar (data not shown). 


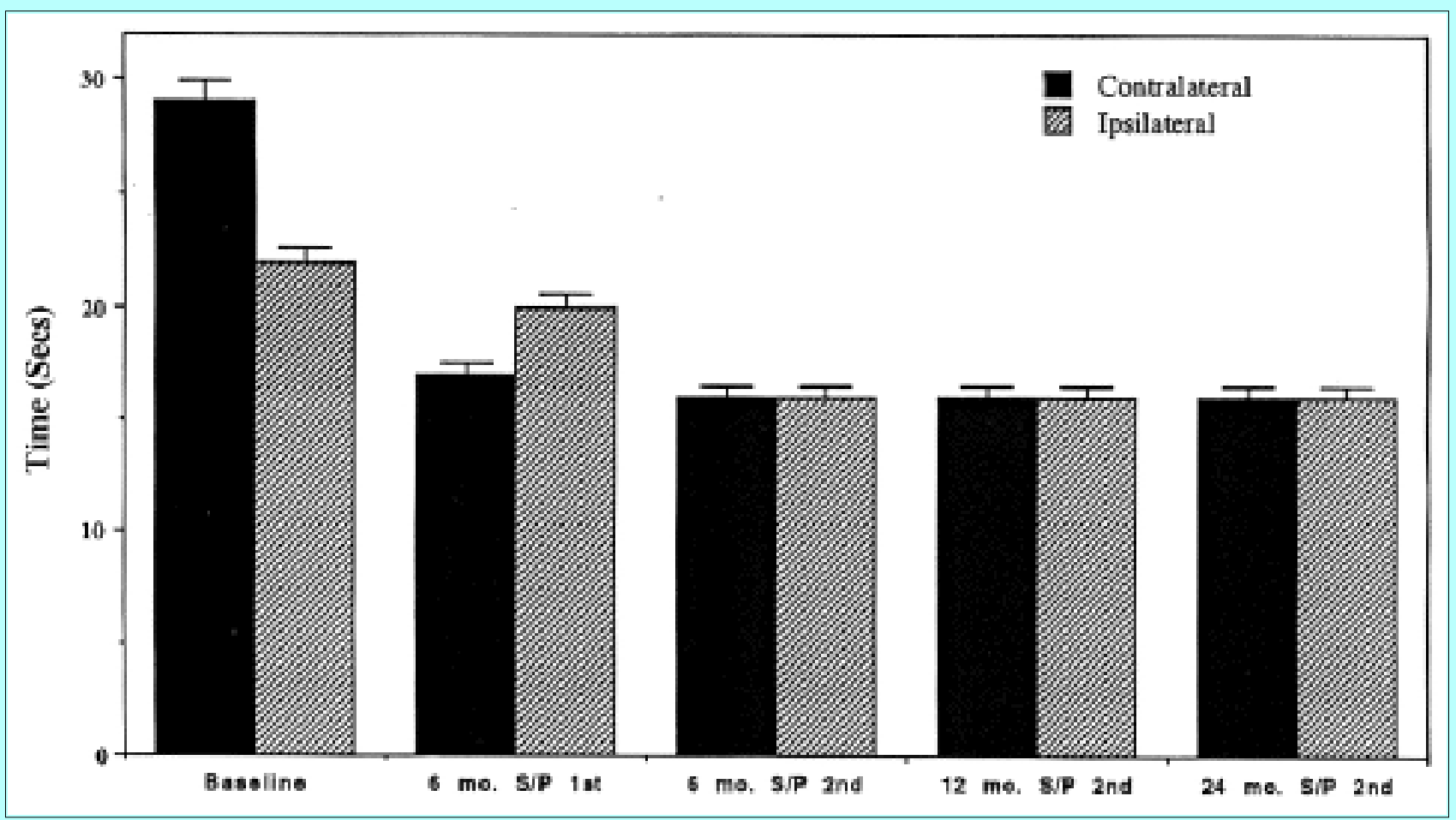

Fig. 2. Bar graph showing mean CAPIT scores following staged bilateral pallidotomy in eight patients. Performance contralateral and ipsilateral to the side of the first procedure are shown.

\section{Subjective Patient Responses}

Of the eight patients who responded to the telephone interview, all stated that they believed the first procedure was beneficial. Four of the eight felt that the second procedure was slightly beneficial and the remaining four felt they derived no benefit. The only symptom that the second procedure improved in all four patients who reported benefit was dyskinesia, with bradykinesia, rigidity, and tremor only slightly improved or not at all.

\section{Postoperative Complications}

Adverse effects of either pallidotomy procedure in this group of patients consisted of worsened speech associated with the second procedure in two cases. In one of these patients with atypical PD and Shy-Drager syndrome the worsened speech was permanent. No complications resulted from his first procedure; however, he experienced akinetic mutism following the second. In the second patient, intraoperative test stimulation consistently produced total speech arrest of approximately 5 minutes duration. Microelectrode recording data in this trajectory were consistent with the correct location within GPi. Because of the observed deficit, lesioning was not performed. The patient was discharged at his neurological baseline, with speech intact.

No other complications were observed and no visual or cognitive deficits were produced.

\section{DISCUSSION}

The clinical improvement following the first pallidotomy in this series of patients is consistent with the growing body of experience with posteroventral pallidotomy. It is clear that unilateral posteroventral pallidotomy, when performed with careful localization and in properly selected patients, can produce excellent improvement in cardinal manifestations of PD such as bradykinesia, rigidity, and 
levodopa-induced dyskinesias.

The role of bilateral pallidotomy, however, is far from clear. Early experiences with bilateral thalamotomies and pallidotomies produced severe complications, raising fears that this option was untenable. Bilateral ventrolateral thalamotomy resulted in aphasia, mutism, quadriparesis, and exacerbation of bradykinesia.[7,13] Bertrand[3] reported two deaths following bilateral posteroventral pallidotomy.

The results of the present study in 12 attempted bilateral pallidotomies suggest that the risk of producing speech deficit with a second procedure may be high. We observed this complication in two patients $(17 \%)$ in our series of attempted bilateral pallidotomies. This finding is difficult to generalize, however. The patient who experienced persistent akinetic mutism had Shy-Drager syndrome and atypical PD. It is possible that with more rigorous selection of patients for bilateral pallidotomy that this complication may be seen less frequently. The other patient experienced transient speech arrest with test stimulation, suggesting that potential speech difficulty might be detectable prior to making a permanent lesion in patients with true idiopathic PD.

In addition to the expected contralateral improvement, our patients generally derived significant benefit on the side ipsilateral to the first pallidotomy, manifested as a $15 \%$ improvement in CAPIT scores. This is consistent with our experience in performing more than 150 pallidotomies at New York University and with the reports by other authors.[4,5,9] In our series, the second pallidotomy added only an additional $10 \%$ improvement in CAPIT and UPDRS scores. The patients also viewed the second procedure as only marginally helpful, with half of the respondents saying that the second lesion did not seem to produce any noticeable improvement. Of those who felt they had benefited from the second procedure, all reported improvement in dyskinesia.

Iacono, et al.,[9] recently reported on 68 patients in whom contemporaneous bilateral pallidotomies were performed as part of a group of 126 patients undergoing pallidotomy. They observed three complications in this subgroup, consisting of two hematomas and one abscess. In addition, their complication rate with contemporaneous bilateral pallidotomies was comparable to that of staged procedures. They concluded that bilateral pallidotomy is well tolerated and that contemporaneous procedures are preferable to staged. Clearly, further experience with this procedure, with continued careful follow up, is needed to clarify this issue.

Based on the results in this group of patients, we believe that bilateral pallidotomy has a narrow therapeutic window. The ipsilateral improvement following the first lesion is augmented only minimally by a second, whereas the risk of speech deficit is greatly enhanced. Patients considered for a second, contralateral procedure should be selected carefully, with disabling dyskinesia being the best indication. We believe that, for the subset of patients who require bilateral procedures, safer alternatives such as chronic pallidal stimulation[15] should be considered. The advantages of pallidal stimulation, most notably its reversibility and the ability to tailor stimulation to the patient's needs and responses, merit further investigation.

\section{References}

1. Alterman RL, Kelly P, Beric A, et al: Selection criteria for unilateral posteroventral pallidotomy. Acta Neurochir (In press, 1997) 
2. Baron MS, Vitek JL, Bakay RE, et al: Treatment of advanced Parkinson's disease by posterior GPi pallidotomy: 1-year results of a pilot study. Ann Neurol 40:355-366, 1996

3. Bertrand CM: A pneumotaxic technique for producing localized cerebral lesions and its use in the treatment of Parkinson's disease. J Neurosurg 15:251-264, 1958

4. Dogali M, Fazzini E, Kolodny E, et al: Effects of posteroventral pallidotomy on Parkinson's disease. Adv Neurol 69:585-590, 1996

5. Dogali M, Fazzini E, Kolodny E, et al: Stereotactic ventral pallidotomy for Parkinson's disease. Neurology 45:753-761, 1995

6. Fahn S, Elton RL, members of the UPDRS Development Committee: Unified Parkinson's disease rating scale, in Fahn S, Marsden CD, Goldstein M, et al (eds): Recent Developments in Parkinson's Disease. New York: MacMillan, 1987, pp 153-163

7. Hassler R, Mundinger F, Riechert T: Stereotaxis in Parkinson Syndrome. New York: Springer-Verlag, 1979, pp 146-248

8. Iacono RP, Lonser RR, Yamada S: Contemporaneous bilateral postero-ventral pallidotomy for early onset "juvenile type" Parkinson's disease. Case report. Acta Neurochir 131:247-252, 1994

9. Iacono RP, Shima F, Lonser RR, et al: The results, indications, and physiology of posteroventral pallidotomy for patients with Parkinson's disease. Neurosurgery 36:1118-1125, 1995

10. Laitinen LV, Bergenheim AT, Hariz MI: Leksell's posteroventral pallidotomy in the treatment of Parkinson's disease. J Neurosurg 76:53-61, 1992

11. Langston JW, Widner H, Goetz CG, et al: Core assessment program for intracerebral transplantations (CAPIT). Mov Disord 7:2-13, 1992

12. Lozano AM, Lang AE, Galvez-Jimenez N, et al: Effect of GPi pallidotomy on motor function in Parkinson's disease. Lancet 346:1383-1387, 1995

13. Narabayashi H: Surgical treatment in the levodopa era, in Stern GM (ed): Parkinson's Disease. Baltimore: Johns Hopkins University Press, 1990, pp 597-646

14. Schaltenbrand G, Bailey P: Introduction to Stereotaxis, With an Atlas of the Human Brain. Stuttgart: Thieme, 1959

15. Siegfried J, Lippitz B: Bilateral chronic electrostimulation of ventroposterolateral pallidum: a new therapeutic approach for alleviating all parkinsonian symptoms. Neurosurgery 35:1126-1130, 1994

Manuscript received January 24, 1997.

Accepted in final form February 6, 1997.

Address reprint requests to: Ron Alterman, M.D., 530 First Avenue, Suite 8R, New York, New York 10016. 